\title{
A wearable haptic feedback system for assisting lower-limb amputees in multiple locomotion tasks
}

\author{
Ilaria Cesini, Giacomo Spigler, Sahana Prasanna, Domitilla Taxis, \\ Filippo Dell'Agnello, Elena Martini, Simona Crea, Nicola Vitiello, \\ Alberto Mazzoni, Calogero Maria Oddo
}

\begin{abstract}
Lower limb prosthesis performance determines the quality of life of amputee patients. Such performance will benefit from a feedback informing the patient about the gait phase and the overall condition of the foot. This study reports the design and validation of a wearable haptic feedback system conceived to assist lower-limb amputees in various locomotion scenarios. Three vibrating motors were attached to a belt in textile to provide information about the foot-ground contact, by remapping the variables detected under the foot, on the waist of the user. Multiple activation patterns were implemented and compared in a pilot study involving one able-bodied subject, during walking, ascending and descending stairs. A novel assessment protocol was proposed to benchmark the different stimulation patterns. The protocol resulted to be a viable method for quicker development and testing of new strategies.
\end{abstract}

\section{INTRODUCTION}

$\mathrm{S}$ ensory information from lower-limbs is crucial for individuals to maintain balance, ambulate and adapt to the physical environment. The main consequences of lower-limb loss are the deterioration of the overall balance performance, decreased gait pattern quality, higher risk of falling and energy consumption [1]. Thus, the restoration of sensory feedback in lower-limb amputees might improve their stability and increase the confidence of the user while using the prosthesis.

Many studies proposed noninvasive systems to compensate for sensory deficit, in which the feedback was delivered through visual [2], auditory [2], [3] or haptic [2], [4], [5] channels, following the principle that the subjects can incorporate the augmented information into their body control scheme in order to restore a more physiological gait pattern [5]. However, most of the proposed solutions focus on ground walking, while little attention has been devoted to exploring other application scenarios, such as ascending and descending stairs.

The goal of this project was to develop a completely wearable haptic feedback device to convey information about the foot-ground interactions by selecting a stimulation strategy out of multiple ones. We developed a protocol to compare the efficacy of the proposed stimulation patterns on intact subjects and to identify the most intuitive protocol for

This work was supported in part by in part by the EU within the CYBERLEGs Plus Plus project (H2020-ICT-2016-1 Grant Agreement \#731931) and in part by the Italian National Institute for Insurance against Accidents at Work (INAIL Centro Protesi, Budrio) within the MOTU project. I. Cesini, G. Spigler, S. Prasanna, F. Dell'Agnello, E. Martini, S. Crea, N. Vitiello, A. Mazzoni, and C. Oddo are with The BioRobotics

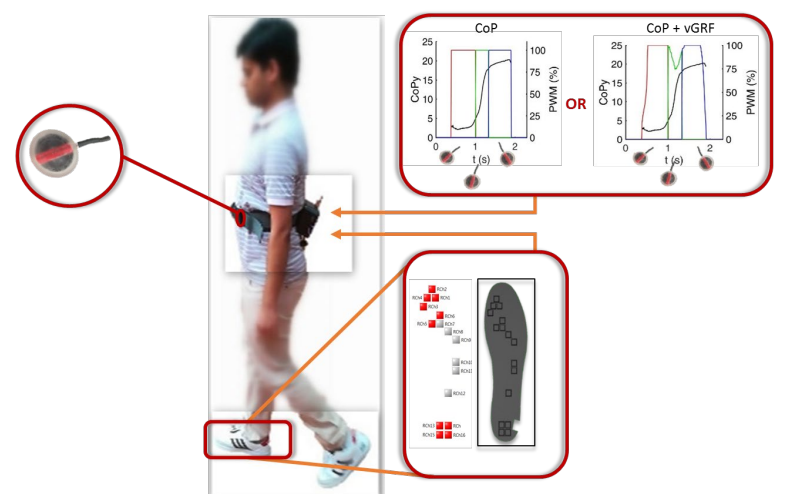

Fig. 1 Outline of the wearable haptic feedback apparatus, consisting of a sensorized insole, a textile belt endowed with vibrotactile units (VTs), and control electronics (VibroMOTUr board).

the amputees. The validation protocol was designed to be adapted to different locomotion tasks, i.e. over-ground walking, ascending and descending stairs.

The present work reports the experimental setup and the preliminary tests conducted with one intact subject to validate the device and the stimulation strategies, and investigate potential criticalities, before extending the feedback system to amputees.

\section{MATERIALS AND METHODS}

\section{A. System setup}

The wearable haptic feedback apparatus was composed of a sensorized interface, driving electronics for data processing and motor control and three vibrating motors (Pico Vibe 304116, Precision Microdrives) attached to a textile belt. In this study, we used a pressure sensitive insole to detect footground interactions. The device integrated an array of optoelectronic sensors and was developed at Scuola Superiore Sant'Anna (Pisa, Italy) [6]. The data acquired from the insole were wirelessly transmitted to a central processing unit, namely the VibroMOTUr board, which was physically attached to the belt housing the vibrotactile (VT) stimulation units. The VibroMOTUr board calculated the vertical Ground Reaction Force (vGRF) and Center of Pressure (CoP) coordinates from the raw sensor signals. The board generated the haptic commands to control the VTs, based on three

Institute, Scuola Superiore Sant'Anna, Pisa, Italy. D. Taxis is with University of Twente, Netherlands.

I. Cesini and C. Oddo are the corresponding authors: ilaria.cesini@santannapisa.it calogero.oddo@santannapisa.it. 
different stimulation strategies, described in Section II.C. The three VTs were equally spaced at $9 \mathrm{~cm}$ on one side of the waist of the subject, from navel to spine to generate ipsilateral perception. The vibrating motors were encapsulated in a polymeric matrix of PDMS (Sylgard ${ }^{\circledR} 184$ Silicone Elastomer, Dow Corning) to provide comfortable contact with the skin and attached to the belt through Velcro fasteners. The configuration of the setup is shown in Fig. 1.

\section{B. Experimental Protocol}

An able-bodied male subject of 38 years, weight $62 \mathrm{~kg}$, height $1.68 \mathrm{~m}$ and foot size $42 \mathrm{EU}$ performed the tasks. The subject was provided with a pair of shoes, with the right shoe embedded with the pressure sensitive insole. The VibroMOTUr board was enclosed in a 3D-printed box that was mounted on the left side of the belt using Velcro fasteners. The VTs were attached to the belt on the right side, i.e. the same side as the sensorized shoe. The subject familiarized with the haptic feedback with an initial setup stage, in which he perceived the intensity of the stimulation while walking freely for 2-3 minutes. The experimental trials consisted in ascending 11 steps followed by a short overground walk, or descending the same track (i.e., walking first, followed by descending the stairs). A different feedback stimulation was provided on each trial, pseudo-randomized among three stimulation strategies so that a single strategy was repeated 5 times each ascending and descending the track (for a total of 30 trials). The time required to complete each trial was measured to estimate the performance. In fact, since sensory feedback is not required for intact subjects to walk confidently, we hypothesized that a poor and un-intuitive feedback strategy might increase their cognitive load, affecting gait speed. During the experiment, the subject was not aware that the durations of the trials were a variable of interest, and he performed each task walking at normal speed.

\section{Vibrotactile stimulation strategies}

Two different stimulation strategies i.e. $C o P$ and CoP_vGRF, were compared against a baseline, CoP_Rand. They are shown in Fig. 2(a). Specifically, in the CoP one of three VTs was activated with maximum intensity when ground contact was detected. The activated VT was chosen by segmenting the $\mathrm{CoP}$ on the right foot into three segments, each associated to a different VT $(\mathrm{VT} 1=\{\mathrm{CoP}<50 \mathrm{~mm}\}$, $\mathrm{VT} 2=\{50 \mathrm{~mm} \leq \mathrm{CoP}<190 \mathrm{~mm}\}, \mathrm{VT} 3=\{190 \mathrm{~mm} \leq \mathrm{CoP}\})$. In the CoP_vGRF a single VT was selected and activated as in $C o P$, but the intensity of the activation was modulated according to the vGRF. CoP_Rand was identical to CoP, but the mapping between the CoP segments and the VTs was randomized every time the CoP moved to a different segment.

\section{RESULTS}

The results collected from the subject are shown in Fig. 2(b). It is possible to observe that a difference was found between the strategies in terms of the time required to complete the tasks especially in ascending the stairs, suggesting that the lesser cognitive load is associated to CoP encoding. These preliminary results show that the proposed

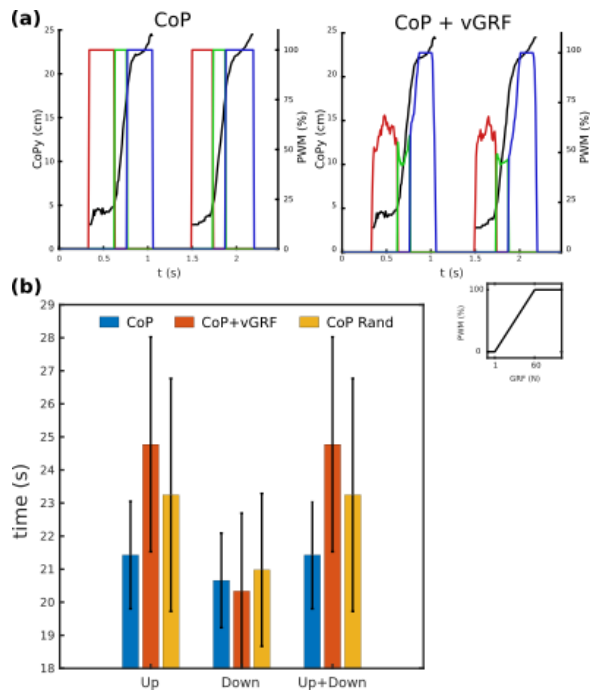

Fig. 2 (a) VTs activation during two steps of walking, using the CoP and CoP_vGRF stimulation strategies. The activation is displayed in red, green and blue, while the center of pressure on the right foot is shown in black. (b) Average time required to walk upstairs (Up) and downstairs (Down) in each protocol, and their combined average (Up+Down). Bars denote standard deviation.

validation protocol is effective in comparing different stimulation strategies.

\section{CONCLUSION}

We presented a new wearable haptic feedback device to convey information about the foot-ground interactions via three VT units, activated according to three different actuation strategies. We further performed a preliminary validation of a novel experimental protocol on one able-bodied subject to compare the stimulation patterns.

Whereas most of the sensory feedback strategies found in literature focus on walking tasks, our stimulation protocol covers different locomotion scenarios. Moreover, we proposed a novel method to assess the performance on intact subjects, which resulted to be viable for selecting the optimal stimulation strategy. The study will be extended to a larger pool of subjects, both intact and amputees.

\section{REFERENCES}

[1] C. Lauretti, G. Pinzari, A. L. Ciancio, A. Davalli, R. Sacchetti, S. Sterzi, E. Guglielmelli, and L. Zollo, "A vibrotactile stimulation system for improving postural control and knee joint proprioception in lower-limb amputees," 2017 26th IEEE Int. Symp. Robot Hum. Interact. Commun., pp. 88-93, 2017.

[2] D. Zambarbieri, M. Schmid, and G. Verni, "Sensory feedback for lower limb prostheses," in Intelligent systems and technologies in rehabilitation engineering, 2001, pp. 129-151.

[3] L. Yang, P. S. Dyer, R. J. Carson, J. B. Webster, K. Bo Foreman, and S. J. M. Bamberg, "Utilization of a lower extremity ambulatory feedback system to reduce gait asymmetry in transtibial amputation gait," Gait Posture, vol. 36, no. 3, pp. 631-634, Jul. 2012.

[4] S. Crea, B. B. Edin, K. Knaepen, R. Meeusen, N. Vitiello, C. Cipriani, M. Donati, M. C. Carrozza, and N. Vitiello, "Time-Discrete Vibrotactile

Feedback Contributes to Improved Gait Symmetry in Patients With Lower Limb Amputations: Case Series," IEEE Trans. Neural Syst. Rehabil. Eng., vol. 23, no. 2, pp. 198-207, 2017.

[5] S. Crea, C. Cipriani, M. Donati, M. C. Carrozza, and N. Vitiello, "Providing Time-Discrete Gait Information by Wearable Feedback Apparatus for Lower-Limb Amputees: Usability and Functional Validation," IEEE Trans. Neural Syst. Rehabil. Eng., vol. 23, no. 2, pp. 250-257, Mar. 2015.

[6] S. Crea, M. Donati, S. De Rossi, C. Oddo, and N. Vitiello, "A Wireless Flexible Sensorized Insole for Gait Analysis," Sensors, vol. 14, no. 1, pp. 1073-1093, Jan. 2014. 